\title{
El mural de Antropología de la UNAN-Managua
}

\author{
Bayardo Gámez Montenegro
}

$\mathrm{E}$ n el año de 1993, la Universidad Nacional Autónoma de Nicaragua, Managua (UNAN-Managua), realizó una serie de cursos especializados en Antropología, los cuales constituyeron la semilla que fructificaría años más tarde a través de la creación y desarrollo del Departamento de Antropología, que hoy cuenta con la carrera de Antropología Social y la maestría en Antropología y Liderazgo Social.

En aquel momento ingresé como participante en el curso de Antropología Urbana impartido por el profesor suizo Bernhardt Albrech del Seminario Etnológico de la Universidad de Zürich y más adelante en el curso de Etnoecología impartido por el profesor polaco Leonardo Tyrtania que venía desde la Universidad Metropolitana de México Iztapalapa.

En este espacio académico se estaban formando los futuros docentes de la carrera de Antropología. Aquí compartí momentos con: Maritza Andino, Gloria
López, María Dolores Álvarez, Mariano Miranda. Unas treinta personas participamos, entre las cuales se encontraban Miriam Díaz, Eduardo Picado, Ruth Aguilar, Auxiliadora Chiong, Silvia Karolina Acuña, Freddy Montenegro, Josefina Hidalgo, Sandra Centeno, Jilma Romero, Miguel Urbina, Yuri Zapata y Alexis White.

Paralelo a estos cursos, estaba organizando y montando la exposición de mis dibujos y pinturas, que titulé Áspero yermo de Tormentas en homenaje al centenario del natalicio del poeta leonés Alfonso Cortés, la cual estuvo abierta al público en la sala de lectura de la Biblioteca Salomón de la Selva de esta misma Alma Mater.

El mural del pabellón 44, en donde se encuentra ubicado el departamento de Antropología de la facultad de Humanidades y Ciencias Jurídicas de la

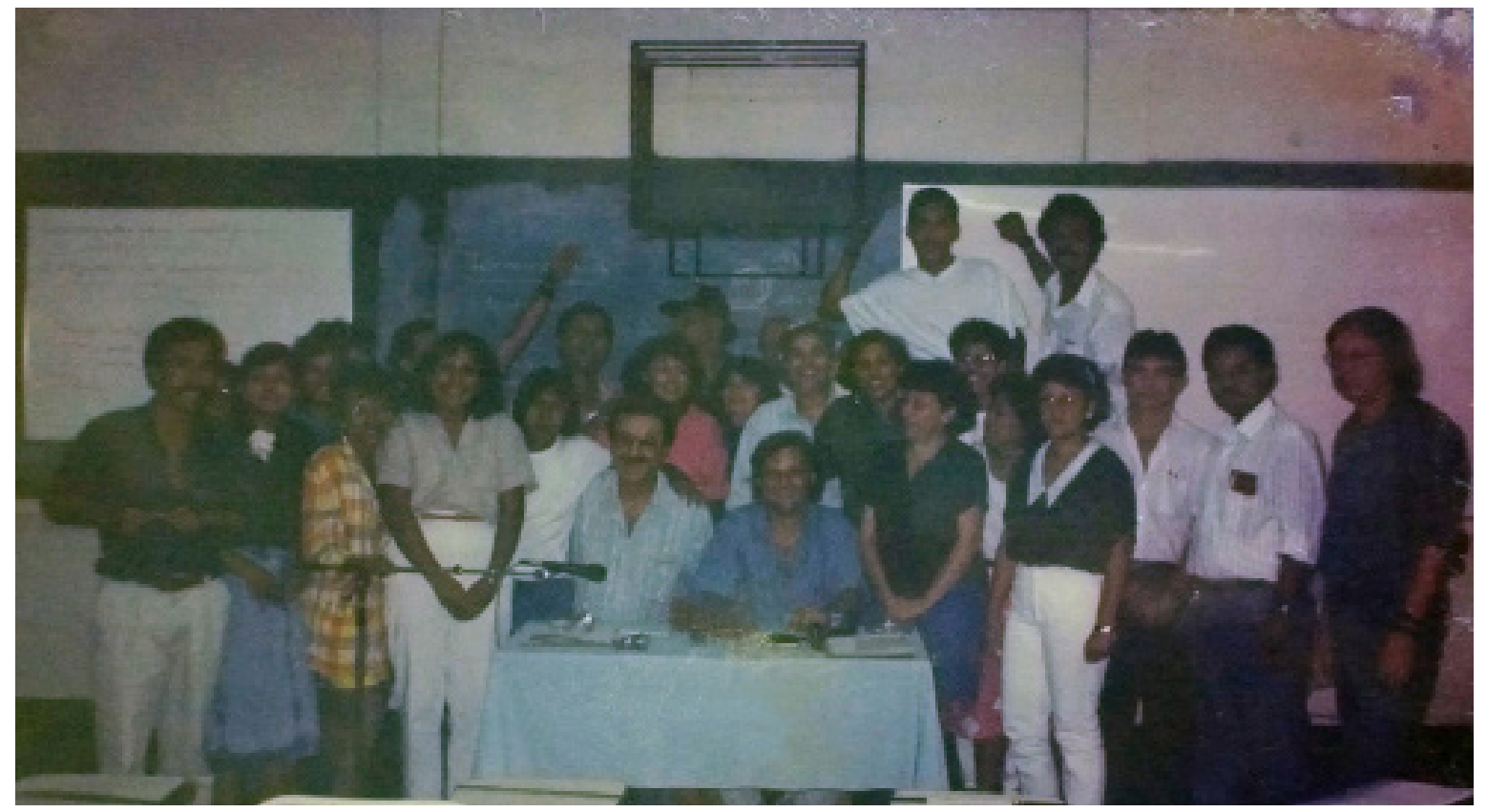

Clausura del curso de Antropología Urbana. UNAN-Managua 1993. 
de la UNAN-Managua, parte de la motivación y agradecimiento público hacia la UNAN-Managua por todo lo aprendido, experiencias compartidas y vividas en diferentes momentos y años.

La idea inicial y luego su realización partió de un diseño creativo que desarrollé para representar el arte, cultura y cosmovisión de los pueblos originarios que han dejado su huella a lo largo y ancho de nuestro territorio nacional.

El primer boceto lo preparé utilizando acuarelas y marcadores sobre cartulina blanca, tomando en cuenta la forma y estructura de la pared rectangular donde se estaría pintando el mural definitivo. Entonces, consideré importante tomar en cuenta la forma geométrica rectangular en su parte principal y triangular hacia arriba siguiendo el perfil del techo de dos aguas, así como el espacio abierto que permite el paso de los es- tudiantes, docentes y personal administrativo hacia los pasillos, oficinas y aulas cercanas.

El concepto surge a partir de la iconografía que se ha publicado en libros, informes de investigación así mismo, el conocimiento directo a través de visitas in situ sobre el arte rupestre nicaragüense, sobre todo lo que han sido los grabados sobre rocas volcánicas de basalto y otras en rocas calcáreas.

Todas las figuras representadas fueron seleccionadas tomando en cuenta su ubicación geográfica y su pertenencia a la diversidad de culturas.

Es así que tenemos figuras tomadas del arte rupestre de la zona Norte, y que están en los sitios de Chagüitillo en Sébaco, Matagalpa y en el sitio de Las Pintadas en Estelí, la región central de Villa Sandino, Chontales.

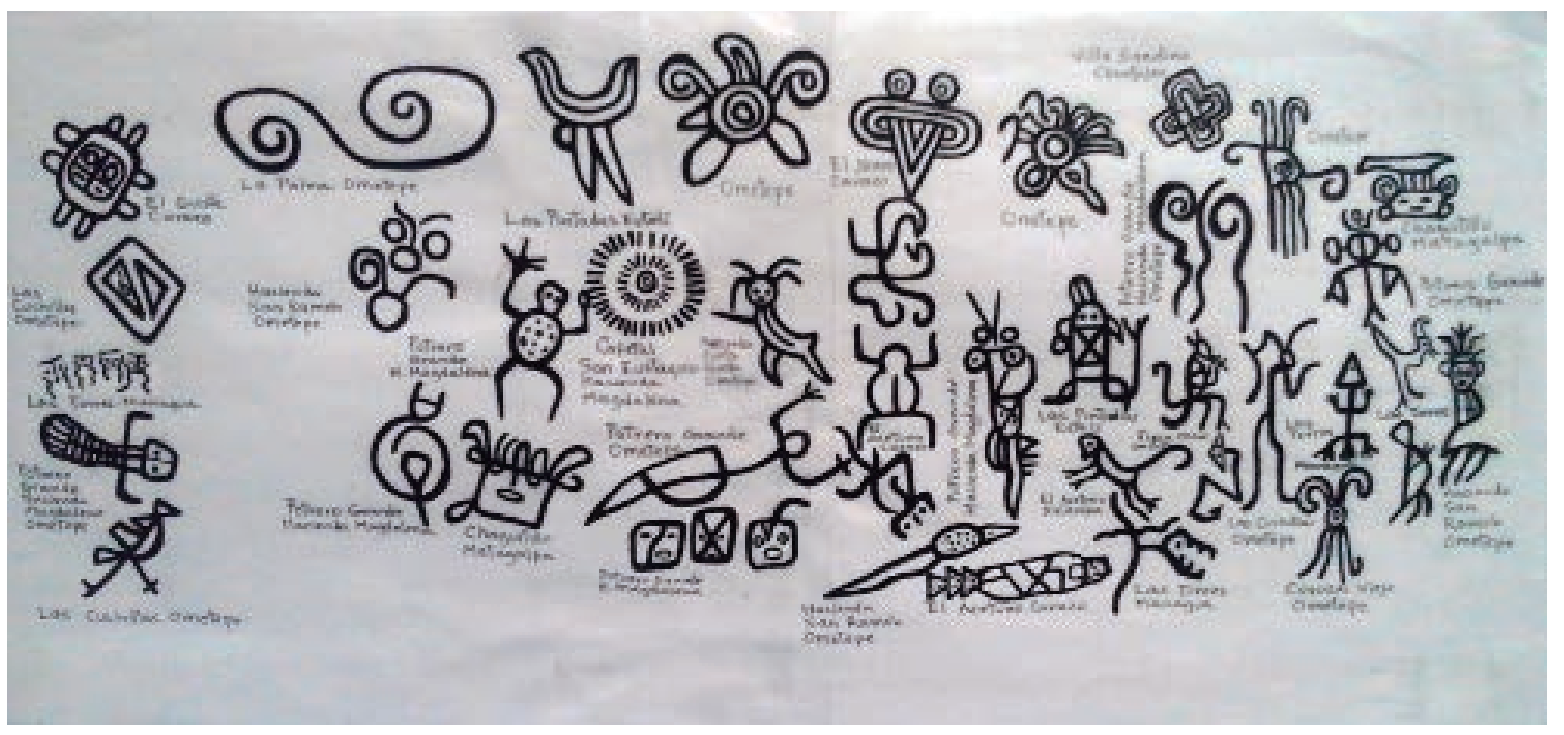

Dibujo a tinta, diseño de mural.

Otras figuras pertenecen a la zona del Pacífico entre las que se encuentran: El Acetuno y El Jícaro en el departamento de Carazo; Las Torres en Managua; los sitios arqueológicos situados al sur en la Isla de Ometepe tales como: Potrero Grande, San Eustaquio, La Palma, Las Cuchillas, Corozal Viejo, Punta Gorda, las haciendas Magdalena y San Ramón.
Se trata de interpretar un tanto la cosmovisión de los nativos nicaragüenses, es una manera de realizar una extracción de raíces culturales a partir del arte rupestre que se encuentra en lugares de la geografía del territorio de Nicaragua con diversas manifestaciones artísticas en petroglifos y pictografías, con una variedad de formas, figuras, temas, simbología y amplitud de significados. 


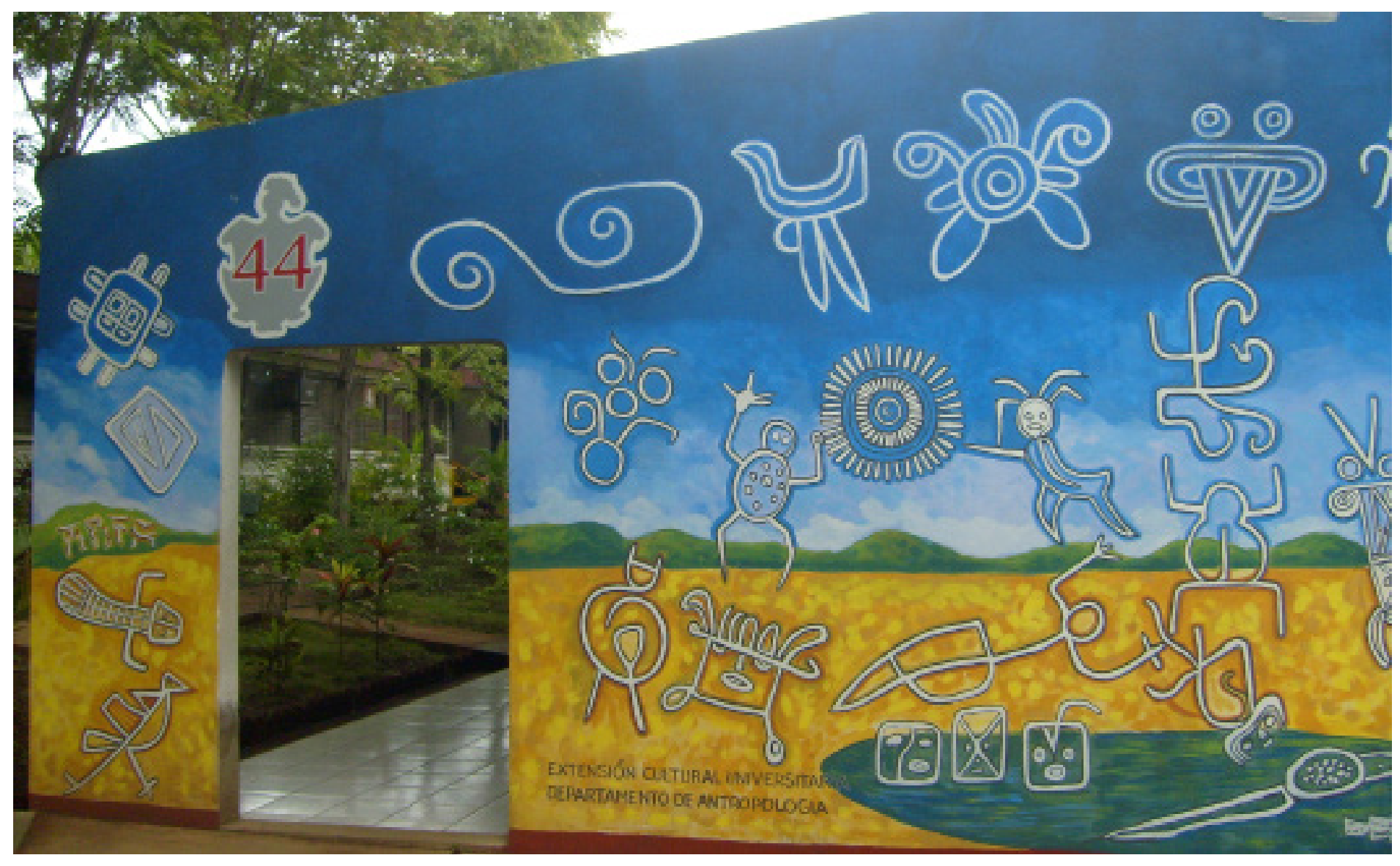

Mural recién pintado, 2007.

Hice un paisaje-escenario organizado en tres franjas: arriba el cielo, en medio el horizonte, abajo la tierra y el agua en forma de lago. Sobre esta base se fueron colocando, diseñando y pintando los diversos gráficos que corresponden a los espacios de cielo como los astros, sol, luna, elementos de la naturaleza como la lluvia, estrellas, orientaciones cardinales, aves y abstracciones geométricas.

En la franje de en medio del horizonte representé las montañas, algunas especies de animales, mamíferos. Abajo se encuentra la tierra con reptiles, insectos, plantas, flores, vegetación, el agua, el ser humano, los curanderos y chamanes para curar las enfermedades físicas y espirituales, y símbolos de los pobladores creados como una forma de representar la relación con la naturaleza, el cosmos y sus deidades.

El mural fue plasmado con pintura acrílica por el pintor nicaragüense Mauricio Solís y su ayudante Juan Márquez, ambos contratados por Extensión Cultural Universitaria en el año 2007. 


\section{Raíces}

Revista Nicaragüense de Antropología. Año 1 No. 1/2017

\section{Bibliografía}

Feltz, Jaime; Setright, Anita; Pérez Leiva, Guillermo; Contreras Zambrana, Celia (1994). Dibujos indígenas en manos de la niñez. Editorial Enlace. Managua, Nicaragua.

Gámez, Montenegro, Bayardo (2004).Registro arqueológico de los petroglifos de la Cuenca del rio Estelí. ADESO Las Segovias, SINSLANI. Estelí, Nicaragua.

María, Hno. Hidelberto. (1965) Estas piedras hablan Editorial Hospicio. León, Nicaragua.

Matillo Vila, Joaquín (Hidelberto María). (1965). Ometepe Isla de círculos y espirales. Centro de investigaciones rupestres. Publicaciones UCA, Managua.

Foto del grupo fundador en 1993 y del mural en 2007, ambas tomadas por Julio Molina de $\mathrm{f} 2.8$.

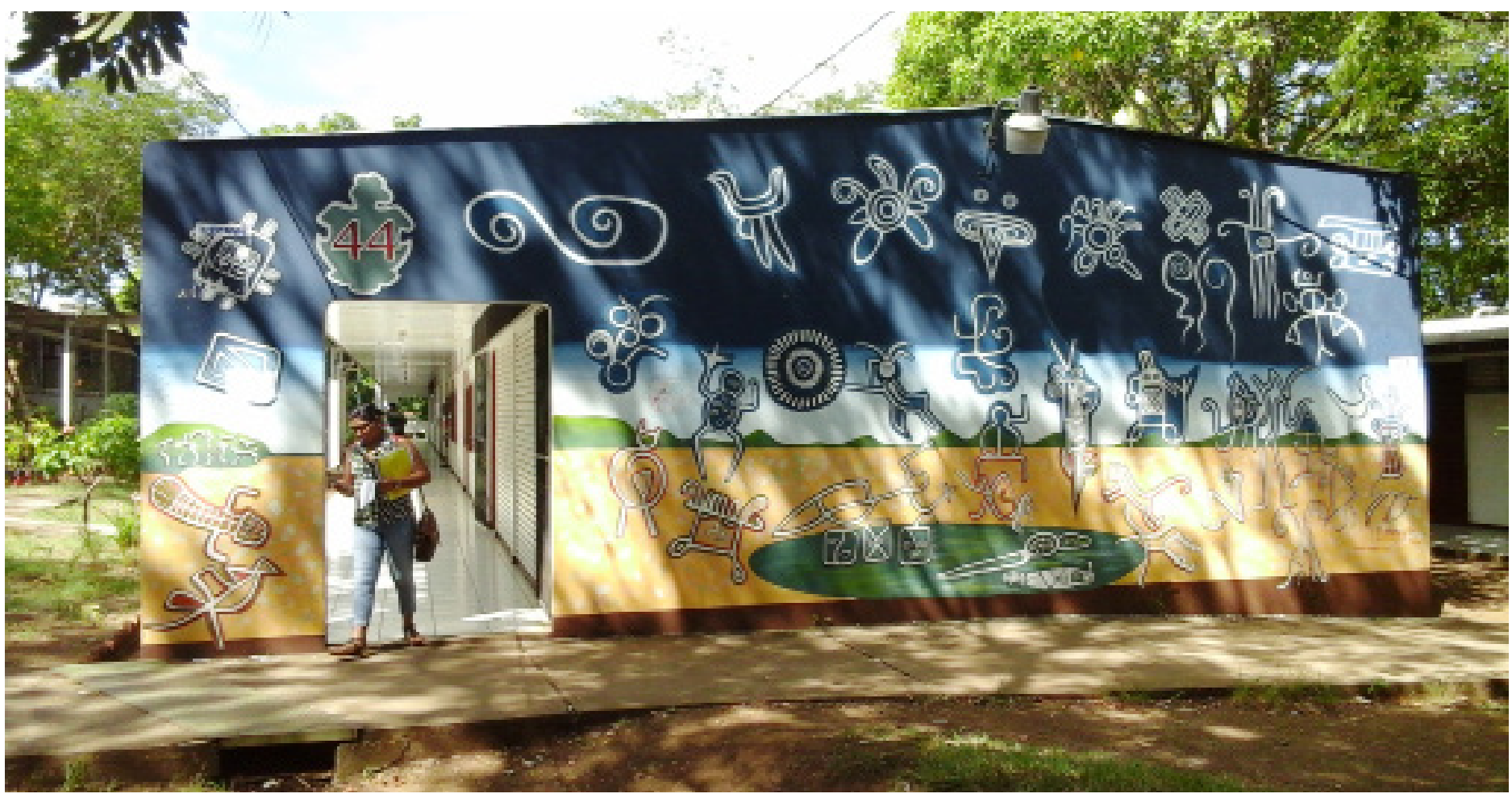

